Laser Chem., 1999, Vol. 19, pp. 109-116 Reprints available directly from the publisher Photocopying permitted by license only
(C) 1999 OPA (Overseas Publishers Association) N.V.

Published by license under

the Harwood Academic Publishers imprint,

part of The Gordon and Breach Publishing Group.

Printed in India.

\title{
COMPLETE DETERMINATION OF RELAXATION PARAMETERS FROM TWO-DIMENSIONAL RAMAN SPECTROSCOPY
}

\author{
VLADIMIR CHERNYAK, ANDREI PIRYATINSKI \\ and SHAUL MUKAMEL* \\ Department of Chemistry, University of Rochester, Rochester, \\ N.Y. 14627, USA
}

(Received 28 June 1998)

\begin{abstract}
Using the model of a weakly-anharmonic, underdamped oscillator coupled to a bath, we demonstrate that the 2D time-resolved Raman signal carries information about the population decay $T_{1}$, the homogeneous dephasing $T_{2}$, and the inhomogeneous dephasing $T_{I}$ relaxation timescales. We distinguish between two projections of the $2 \mathrm{D}$ signal: first, the echo signal, which is stretched in the diagonal direction decays with $T_{2}$, and the second which is stretched along one axis is related to the population relaxation and decays with $T_{1}$. The width of both signals reflects $T_{I}$. Equations of motion for vibrational coherence and population variables are employed in these calculations.
\end{abstract}

Keywords: Femtosecond spectroscopy; photon echo; multidimensional Raman spectroscopy; pure dephasing

The advent of femtosecond lasers with pulse durations under $10 \mathrm{fs}$ has opened recently a new field of impulsive Raman vibrational spectroscopy [1,2]. Such pulses are short compared with vibrational frequencies even for high-frequency $\sim 2000 \mathrm{~cm}^{-1}$ vibrations. This allows to excite vibrational modes impulsively and observe coherent oscillations in real time. Besides oscillations, optical signals are damped due to coupling to lower-frequency bath modes, e.g., intermolecular, solvent, etc., and the signals carry important informa-

\footnotetext{
*Corresponding author.
} 
tion on the system-bath coupling. Conventional CARS spectroscopy is based on the third-order optical response which is one-dimensional (1D) and the resulting information is highly averaged. Higher-order techniques e.g., 2D Raman spectroscopy based on the fifth-order optical response provide much more detailed information [3-6]. For underdamped modes whose frequencies are larger than the linewidths $\Delta$, one can clearly distinguish between three line broadening mechanisms contributing to the 1D signal decay rate: homogeneous dephasing, inhomogeneous dephasing and finite lifetime. The inhomogeneous dephasing timescale $T_{I}$ is induced by static disorder resulting from coupling to low frequency modes. Homogeneous dephasing $\left(T_{2}\right)$ corresponding to fast bath modes consists of pure dephasing $\left(T_{2}^{*}\right)$ processes which only destroy vibrational coherence and lifetime $\left(T_{1}\right)$ representing vibrational population relaxation $1 / T_{2}=1 / T_{1}+1 / T_{2}^{*}$. These three mechanisms result in the $T_{I}, T_{2}$ and $T_{1}$ relaxation timescales. CARS spectroscopy provides information on the overall value of $1 / T_{2}+1 / T_{1}+1 / T_{2}^{*}$. The capacity of $2 \mathrm{D}$ Raman spectroscopy to distinguish between the homogeneous and inhomogeneous dephasing using the photon echo approach has been studied [7]. In this paper we demonstrate that a single 2D measurement can yield all three timescales.

In impulsive Raman spectroscopy the system remains in the ground electronic state at all times and during the time intervals between pulses the evolution is determined by the vibrational Hamiltonian of the ground electronic state. The coupling to the driving field can be described by the electronic polarizability $\alpha(\mathbf{Q})$ which depends parametrically on the set of nuclear coordinates denoted by $\mathbf{Q}[8]$. The 1D heterodyne detected impulsive signal is given by the two-point Liouville space correlation function

$$
S^{(1 \mathrm{D})}\left(t_{1}\right)=\left\langle\alpha_{+}\left(t_{1}\right) \alpha_{-}(0)\right\rangle,
$$

where for any operator $A$ in the Hilbert space, $A_{ \pm}$denote Liouville space superoperators defined by the following action on the density matrix $\rho: A_{-} \rho=[A, \rho], A_{+}=(A \rho+\rho A) / 2$ [9]. The 2D signal is expressed in terms of a three point correlation function [9]

$$
S^{(2 \mathrm{D})}\left(t_{2}, t_{1}\right)=\left\langle\alpha_{+}\left(t_{2}+t_{1}\right) \alpha_{-}\left(t_{1}\right) \alpha_{-}(0)\right\rangle .
$$


We consider a system with a single primary anharmonic vibration with the Hamiltonian

$$
\begin{aligned}
H & =H_{0}+H_{1}, \\
H_{0} & =\frac{P^{2}}{2 M}+\frac{M \Omega^{2} Q^{2}}{2}, \quad H_{1}=\sum_{j=3}^{\infty} \frac{Q^{j}}{j !} V^{(j)},
\end{aligned}
$$

where $H_{0}$ and $H_{1}$ represent the harmonic and anharmonic parts respectively. The primary mode is coupled to a bath described by the bath Hamiltonian which depends on a set of bath coordinates q. The coupling is described by the system-bath interaction Hamiltonian $H_{\text {int }}$. We further assume, that the polarization operator $\alpha$ only depends on the primary Raman-active mode coordinate:

$$
\alpha(Q)=\alpha^{(1)} Q+\sum_{j=2}^{\infty} \alpha^{(j)} \frac{Q^{j}}{j !} .
$$

In the limit of weak nonlinearity [9] the main contribution to the 2D signal is given by the terms which are first order in $\alpha^{(2)}$ or $V^{(3)}$. This yields three contributions of the form:

$$
\begin{aligned}
& S_{1}\left(t_{2}, t_{1}\right)=\alpha^{(2)} \alpha^{(1)} \alpha^{(1)}\left\langle Q_{+}\left(t_{2}+t_{1}\right) Q_{+}\left(t_{1}\right) Q_{-}\left(t_{1}\right) Q_{-}(0)\right\rangle, \\
& S_{2}\left(t_{2}, t_{1}\right)=\alpha^{(2)} \alpha^{(1)} \alpha^{(1)}\left\langle Q_{+}\left(t_{2}+t_{1}\right) Q_{-}\left(t_{1}+t_{2}\right) Q_{-}\left(t_{1}\right) Q_{-}(0)\right\rangle, \\
& S_{3}\left(t_{2}, t_{1}\right)=\alpha^{(1)} \alpha^{(1)} \alpha^{(1)} \frac{V^{3}}{3 !} \int_{0}^{t_{2}} d \tau\left\langle Q_{+}\left(t_{2}+t_{1}\right)\left[Q^{3}(\tau)\right]_{-} Q_{-}\left(t_{1}\right) Q_{-}(0)\right\rangle,
\end{aligned}
$$

and the expectation values in Eqs. (5) are taken with respect to $H_{0}$. If the bath is harmonic and the system-bath interaction is linear in the primary and bath coordinates, the correlation functions in Eqs. (5) can be evaluated exactly $[9,10]$. However this type of system-bath interaction describes lifetime effects only, and cannot represent pure dephasing processes. These can only be described by adding anharmonicities, e.g., $Q^{2} q$ terms. In this case the correlation functions in Eqs. (5) may not be computed exactly. They can be, however, calculated by eliminating the bath using projection operator techniques [11] and calculating the necessary kernels to second order in the system-bath coupling. An alternative and more intuitive way is to use 
equations of motion for the primary variables, adopting a factorization scheme and eliminating the bath using projection operators. It has been shown [9] that in the case of linearly coupled harmonic bath, the expressions given by Eqs. (5) can be calculated using an equation of motion for the $\langle Q\rangle$ variable only, by factorizing the higher-order products using $\left\langle Q^{j}\right\rangle=\langle Q\rangle^{j}$. This is a classical approximation [9] and, in the theory of Frenkel exciton systems this is known as the Local Field Approximation (LFA). The LFA fails to describe pure dephasing processes: to this end additional population variables should be included $[12,13]$.

To derive a closed system of equations for vibrations which is similar to that in Refs. [12, 13] we first express the vibrational coordinate $Q$ and momentum $P$ in terms of the Boson creation (annihilation) operators $\hat{B}^{\dagger}(\hat{B})$

$$
Q=\frac{1}{\sqrt{2 M \Omega}}\left(\hat{B}^{\dagger}+\hat{B}\right), \quad P=i \sqrt{\frac{M \Omega}{2}}\left(\hat{B}^{\dagger}-\hat{B}\right) .
$$

We further retain only the cubic term in the anharmonicity Hamiltonian Eq. (3): $H_{1}=\frac{1}{3 !} V^{(3)} Q^{3}$ and add an effective coupling between the primary oscillator and electronically off-resonant radiation field $E(t): \quad H_{\text {eff }}=\alpha(Q) E^{2}(t)$. After the expansion of the polarization operator $\alpha(Q)$ (Eq. (4)) to second order in $Q$ and substitution of Eq. (6) the Hamiltonian of the system coupled to the driving field becomes:

$$
H_{T}=\Omega \hat{B}^{\dagger} \hat{B}+\frac{\tilde{V}^{(3)}}{3 !}\left(\hat{B}^{\dagger}+\hat{B}\right)^{3}-\left(\tilde{\alpha}^{(1)}\left(\hat{B}^{\dagger}+\hat{B}\right)+\frac{\tilde{\alpha}^{(2)}}{2 !}\left(\hat{B}^{\dagger}+\hat{B}\right)^{2}\right) E^{2}(t)
$$

where we use the notation $\tilde{V}^{(3)}=V^{(3)} /(2 M \Omega)^{3 / 2}$, and $\tilde{\alpha}^{(j)}=$ $\alpha^{(j)} /(2 M \Omega)^{j / 2}$. The radiation field $E(t)$ is represented by a sequence of two pairs of ultrashort non-overlapping pulses centered at times $\tau_{1}>\tau_{2}$, respectively and followed be the probe pulse $\mathcal{E}_{p}$ :

$$
E(t)=\sum_{n=1}^{2} \mathcal{E}_{n}\left(t-\tau_{n}\right)+\mathcal{E}_{p}(t)
$$

The delay times between pulses $t_{1}=\tau_{2}-\tau_{1}$ and $t_{2}=t-\tau_{2}$ were used in Eqs. (5). 
Taking into account homogeneous dephasing and population decay requires equations of motion for two observables $B(t) \equiv\langle\hat{B}(t)\rangle$ (and its complex conjugate $\left.B^{*}(t) \equiv\left\langle\hat{B}^{\dagger}\right\rangle\right)$ and $n(t) \equiv\left\langle\hat{B}^{\dagger} \hat{B}\right\rangle$. Making use of the Heisenberg equation of motion for the operators $\hat{B}, \hat{B}^{\dagger} \hat{B}$ and applying the following factorization procedure: $\left\langle\hat{B}^{\dagger} \hat{B}^{\dagger}\right\rangle=\left\langle\hat{B}^{\dagger}\right\rangle\left\langle\hat{B}^{\dagger}\right\rangle$, $\langle\hat{B} \hat{B}\rangle=\langle\hat{B}\rangle\langle\hat{B}\rangle$ we obtain:

$$
\begin{aligned}
\dot{B}= & \left(-i \Omega-\frac{\Gamma}{2}\right) B-i \frac{\tilde{V}^{(3)}}{2}\left(B^{* 2}+B^{2}+2 N\right) \\
& +i\left(\tilde{\alpha}^{(1)}+\tilde{\alpha}^{(2)}\left(B^{*}+B\right)\right) E^{2}(t), \\
\dot{N}= & -\gamma N+i \tilde{\alpha}^{(1)}\left(B^{*}-B\right) E^{2}(t),
\end{aligned}
$$

where $N=n(t)-n_{\text {eq }}, n_{\text {eq }}$ is the vibrational population at thermal equilibrium. In the r.h.s. of Eqs. (9) we retain only terms up to $Q^{2}$ and take into account the anharmonic renormalization of the vibrational frequency $\Omega$. We also introduce relaxation through the dephasing rate $\Gamma=\gamma+\hat{\Gamma}=2 / T_{2}$ and population decay $\gamma=1 / T_{1}, \hat{\Gamma}$ being the pure dephasing rate. The induced polarization which determines the optical signal has the following form:

$$
P=\left(\tilde{\alpha}^{(1)}\left(B^{*}+B\right)+\frac{\tilde{\alpha}^{(2)}}{2 !}\left(B^{* 2}+B^{2}+2 N\right)\right) E_{p}
$$

By solving Eqs. (9) perturbatively in the driving field and substituting the results into Eq. (10) we obtain the $2 \mathrm{D}$ optical signal. The signal can be represented by its coherent and incoherent components. The former can be obtained by factorizing the population variables in the form $N=B^{*} B$ which is equivalent to the LFA. This implies that the coherent component is given by the expressions derived in $[9,10]$ provided pure dephasing is added to the decay rate. The incoherent component is conveniently represented in terms of the irreducible part of the population defined by $N^{i} \equiv N-B^{*} B$. The first contribution to the signal $S_{1}$ in Eqs. (5) contains the coherent component only. The incoherent components corresponding to the terms $S_{2}, S_{3}$ have the form:

$$
\begin{aligned}
& S_{2}^{i}\left(t_{1}, t_{2}\right)=\tilde{\alpha}^{(2)} N^{i}\left(t_{1}, t_{2}\right), \\
& S_{3}^{i}\left(t_{1}, t_{2}\right)=\tilde{\alpha}^{(1)} \tilde{V}^{(3)} \int_{0}^{t_{2}} d t^{\prime} \sin \left[\Omega\left(t_{2}-t^{\prime}\right)\right] e^{-\frac{\Gamma}{2}\left(t_{2}-t\right)} N^{i}\left(t_{1}, t_{2}-t^{\prime}\right),
\end{aligned}
$$


$N^{i}$ can be evaluated solving Eqs. (9) which yields

$$
N^{i}=2 \tilde{\alpha}^{(1)} \tilde{\alpha}^{(1)} F_{1} F_{2} \cos \left(\Omega t_{1}\right) e^{-\frac{\Gamma t_{1}}{2}}\left(e^{-\gamma t_{2}}-e^{-\Gamma t_{2}}\right),
$$

where $F_{j}=\int_{-\infty}^{\infty} d \tau\left|\mathcal{E}_{j}^{2}(\tau)\right|, j=1,2$. It should be pointed out that in the absence of pure dephasing $(\hat{\Gamma}=0) \Gamma=\gamma$ and $N^{i}$ vanishes, and Eqs. (9) and (10) become equivalent to the LFA [14].

We have solved Eqs. (9) and (10) and averaged the signal over an inhomogeneous Gaussian distribution of vibrational frequencies $W(\Omega)$ with central frequency $\Omega_{0}$ and width $\Delta$. The final expression for the 2D Raman signal is:

$$
\begin{aligned}
S^{(5)}\left(t_{1}, t_{2}\right) \propto & \cos \left[\Omega_{0}\left(t_{2}-t_{1}\right)+\theta\right] e^{-\frac{\Delta^{2}\left(t_{1}-t_{2}\right)^{2}}{2}} e^{-\frac{\Gamma}{2}\left(t_{1}+t_{2}\right)} \\
& +A \cos \left(\Omega_{0} t_{1}\right) e^{-\frac{\Delta^{2} t_{1}^{2}}{2}} e^{-\frac{\Gamma}{2} t_{1}-\gamma t_{2}}
\end{aligned}
$$

Equation (13) is the central result of this paper. The signal consists of two components. The first represents an echo which appears at $t_{1}=t_{2}$ and is associated with the excitation of vibrational coherences, and decays on the homogeneous dephasing timescale $T_{2}=(\Gamma / 2)^{-1}$. The echo signal also shows oscillations with period $T=\left(\Omega_{0} / 2\right)^{-1}$. The oscillations phase shift $\theta$ reflects the weak anharmonicity and is determined by $\tan \theta=\left(\tilde{V}^{(3)} \tilde{\alpha}^{(1)}\right) /\left(\Omega_{0} \tilde{\alpha}^{(2)}\right)$. The second term in Eq. (13) is stretched along the $t_{2}$ direction at $t_{1}=0$ and decays on the population decay timescale $T_{1}=\gamma^{-1}$. This signal also shows oscillations along $t_{1}$ with the period $T=\left(\Omega_{0} / 2\right)^{-1}$. However, in this case the anharmonicity affects the signal intensity (via the parameter $A=1+\tan \theta)$ rather than the oscillations phase. The width of both signals reflects the inhomogeneous dephasing timescale $T_{I}=\Delta^{-1}$. In Figure 1 we present $2 \mathrm{D}$ contour plots of the signal $\left|S^{(5)}\right|^{2}$ computed Eq. (13) using and plotted on the timescale comparable with $\left(T_{1}, T_{2}\right)$. The echo component in the direction $t_{1}=t_{2}$ and the component associated with the population decay stretched along the $t_{2}$-axis at $t_{1}=0$ are clearly seen. Since typically pure dephasing is faster than population relaxation the component stretched along the $t_{2}$-axis decays on a longer timescale compared to the echo component. The oscillations of the signal are clearly shown in the insert which displays the same signal but on the timescale comparable with $\Omega_{0}^{-1}$. 


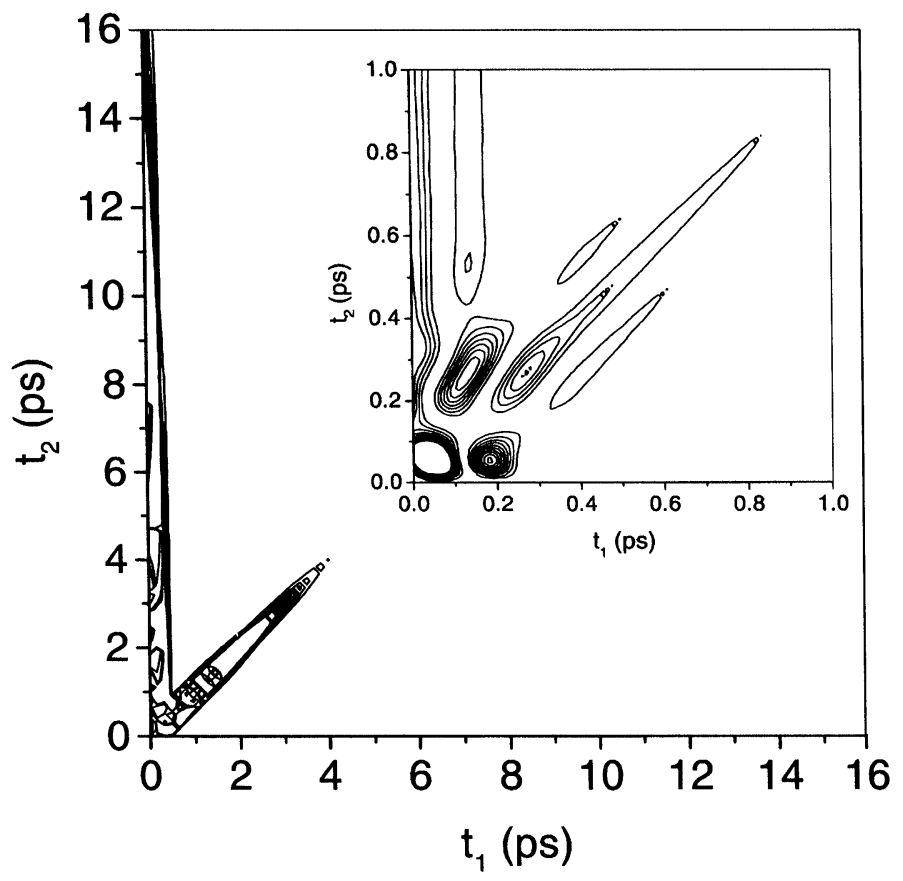

FIGURE 1 Contour plot of the $2 \mathrm{D}$ signal $\left|S\left(t_{2}, t_{1}\right)\right|^{2}$ for a harmonic system $\left(\tilde{V}^{(3)}=0\right.$ in Eq. (13)) coupled to a bath with homogeneous dephasing rate $\Gamma=2.5 \mathrm{~cm}^{-1}$ and population decay rate $\gamma=1 \mathrm{~cm}^{-1}$ averaged over Gaussian frequency distribution with central frequency $\Omega_{0}=112 \mathrm{~cm}^{-1}$ and inhomogeneous width $\Delta=30 \mathrm{~cm}^{-1}$. The echo component in the direction $t_{2}=t_{1}$ decays on the $T_{2}=(\Gamma / 2)^{-1}$ timescale, the component stretched along $t_{2}$-axis at $t_{1}=0$ decays on the population decay timescale $T_{1}=\gamma^{-1}$. The width of both components reflects inhomogeneous dephasing timescale $T_{I}=\Delta^{-1}$. The insert shows the signal oscillations with period $T=\left(\Omega_{0} / 2\right)^{-1}$.

In summary, usually $T_{2}$ is measured by photon echoes, $T_{1}$ is obtained by pump probe [15] and the total linewidth depends on $T_{1}, T_{2}$ and $T_{I}$. We have demonstrated that a single $2 \mathrm{D}$ impulsive Raman echo measurement can provide the complete information about all relaxation parameters $T_{I}, T_{1}$ and $T_{2}$ of a weakly anharmonic vibrational system.

\section{Acknowledgements}

We gratefully acknowledge the support of the National Science Foundation and the United States Air Force Office of Scientific Research. 


\section{References}

[1] Weiner, A. M., Leird, D. E., Weiderrecht, G. P. and Nelson, K. A. (1990). Science, 247, 1317.

[2] Lotshaw, W. T., McMorrow, D., Thantu, N., Melinger, J. S. and Kitchenham, R. (1995). J. Raman Spectrosc., 26, 571.

[3] Tanimura, Y. and Mukamel, S. (1993). J. Chem. Phys., 99, 9496; Khidekel, V. and Mukamel, S. (1995). Chem. Phys. Lett., 240, 304.

[4] Okumura, K. and Tanimura, Y. (1997). Chem. Phys. Lett., 278, 175.

[5] Tokmakoff, A., Lang, M. J., Larsen, D. S., Fleming, G. R., Chernyak, V. and Mukamel, S. (1997). Phys. Rev. Lett., 79, 2702.

[6] Tominaga, K. and Yoshihara, K. (1997). Phys. Rev. A, 55, 831.

[7] Palese, S., Mukamel, S., Miller, R. J. D. and Lotshaw, W. T. (1996). Phys. Chem., $100,10380$.

[8] Hellwarth, R. W. (1977). Progr. Quant. Electron., 5, 2.

[9] Chernyak, V. and Mukamel, S. (1998). J. Chem. Phys., 108, 5812.

[10] Mukamel, S., Piryatinski, A. and Chernyak, V., Acct. Chem. Res. (in press).

[11] Zwanzig, R. (1961). Lect. Thoer. Phys., 3, 106; Physics, 30, 1109, (1964); Mori, H. (1965). Prog. Theor. Phys., 33, 423; 34, 399.

[12] Knoester, J. and Mukamel, S. (1991). Phys. Rep., 205, 1.

[13] Chernyak, V., Zhang, W. M. and Mukamel, S., J. Chem. Phys., (in press); Zhang, W. M., Chernyak, V. and Mukamel, S., Ultrafast Phenomena XI, Zinth, W. and Fujimoto, J. G. Eds. (Springer-Verlag, Berlin) (1998).

[14] Mukamel, S. (1995). Principles of Nonlinear Optical Spectroscopy (Oxford University Press, New York).

[15] Rektor, K. D., Kwok, A. S., Ferrante, C., Tokmakoff, A., Rella, C. W. and Fayer, M. D. (1997). J. Chem. Phys., 106, 10027. 